\title{
Additive maps on prime and semiprime rings with involution
}

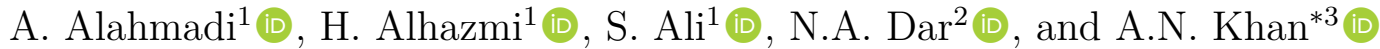 \\ ${ }^{1}$ Department of Mathematics, Faculty of Science, King Abdulaziz University, Jeddah-21589, Saudi Arabia \\ ${ }^{2}$ Department of Computer science and Engineering, IUST, Awantipora, J \& K-192301, India \\ ${ }^{3}$ Department of Mathematics, Faculty of Science $\&$ Arts- Rabigh, King Abdulaziz University, Saudi Arabia
}

\begin{abstract}
Let $R$ be an associative ring. An additive map $x \mapsto x^{*}$ of $R$ into itself is called an involution if (i) $(x y)^{*}=y^{*} x^{*}$ and (ii) $\left(x^{*}\right)^{*}=x$ hold for all $x \in R$. The main purpose of this paper is to study some additive mappings on prime and semiprime rings with involution. Moreover, some examples are given to demonstrate that the restrictions imposed on the hypothesis of the various results are not superfluous.
\end{abstract}

Mathematics Subject Classification (2010). 16N60, 16W10, 16W25

Keywords. prime ring, semiprime ring, normal ring, involution, left $*$-centralizer, Jordan left $*$-centralizer, generalized derivation

\section{Notations and Introduction}

Throughout the present paper, $R$ will represent an associative ring with center $Z(R)$. As usual, the commutator $x y-y x$ will be denoted by $[x, y]$. Given an integer $n \geq 2$, a ring $R$ is said to be $n$-torsion free if $n x=0$ (where $x \in R$ ) implies that $x=0$. A ring $R$ is called prime if $a R b=(0)$ (where $a, b \in R$ ) implies $a=0$ or $b=0$ and is called semiprime ring if $a R a=(0)$ (where $a \in R$ ) implies $a=0$. An additive map $x \mapsto x^{*}$ of $R$ into itself is called an involution if (i) $(x y)^{*}=y^{*} x^{*}$ and (ii) $\left(x^{*}\right)^{*}=x$ hold for all $x \in R$. A ring equipped with an involution is called ring with involution or *-ring. An element $x$ in a ring with involution is said to be Hermitian if $x^{*}=x$ and skew-Hermitian if $x^{*}=-x$. The sets of all Hermitian and skew-Hermitian elements of $R$ will be denoted by $H(R)$ and $S(R)$, respectively. The involution is said to be of the first kind if $Z(R) \subseteq H(R)$, otherwise it is said to be of the second kind. In the latter case, $S(R) \cap Z(R) \neq(0)$. If $R$ is 2 -torsion free then every $x \in R$ can be uniquely represented in the form $2 x=h+k$ where $h \in H(R)$ and $k \in S(R)$. Note that in this case $x$ is normal, i.e., $x x^{*}=x^{*} x$, if and only if $h$ and $k$ commute. If all elements in $R$ are normal, then $R$ is called a normal ring. An example is the ring of quaternions. A description of such rings can be found in [17] and [20], where further references can be found.

\footnotetext{
*Corresponding Author.

Email addresses: adelnife2@yahoo.com (A. Alahmadi), alhazmih@yahoo.com (H. Alhazmi), shakir50@rediffmail.com (S. Ali), ndmdarlajurah@gmail.com (N.A. Dar),

abdulnadimkhan@gmail.com (A.N. Khan)

Received: 06.03.2018; Accepted: 23.08.2019
} 
Let $R$ be a ring. An additive mapping $d: R \rightarrow R$ is called a derivation (respectively reverse derivation) if $d(x y)=d(x) y+x d(y)$ (respectively $d(x y)=d(y) x+y d(x)$ ) holds for all $x, y \in R$. A derivation $d$ is called inner if there exists $a \in R$ such that $d(x)=[a, x]$ for all $x \in R$. According to [16], an additive mapping $d: R \longrightarrow R$ is called a Jordan derivation if $d\left(x^{2}\right)=d(x) x+x d(x)$ holds for all $x \in R$. An additive mapping $F: R \longrightarrow$ $R$ is called a generalized derivation (respectively generalized Jordan derivation) if there exists a derivation (respectively Jordan derivation) such that $F(x y)=F(x) y+x d(y)$ (respectively $F\left(x^{2}\right)=F(x) x+x d(x)$ ) holds for all $x, y \in R$. Obviously, any derivation is a generalized derivation, but the converse is not true in general. A significant example is a map of the form $G(x)=a x+x b$ for some $a, b \in R$; such generalized derivations are called inner. Generalized derivations have been primarily studied on operator algebras. Therefore, any investigation from the algebraic point of view might be interesting (see for example [18] and [19]). In [19], Lee extended the definition of generalized derivation as follows: by a generalized derivation he means an additive mapping $G: J \longrightarrow U$ such that $G(x y)=G(x) y+x d(y)$ for all $x, y \in J$, where $U$ is the left Utumi quotient ring of $R, J$ is a dense right ideal of $R$, and $d$ is a derivation from $J$ to $U$. He also showed that every generalized derivation can be uniquely extended to a generalized derivation of $U$. In fact, there exists $a \in U$ and a derivation $d$ of $U$ such that $G(x)=a x+d(x)$ for all $x \in U$. Considerable work has been done on generalized derivations in prime and semiprime rings during the last few years (see [9-12,15,25,28], where further references can be found).

An additive mapping $T: R \rightarrow R$ is said to be a left centralizer (respectively reverse left centralizer) if $T(x y)=T(x) y$ (respectively $T(x y)=T(y) x)$ for all $x, y \in R$. The definition of a right centralizer (respectively reverse right centralizer) should be self-explanatory. An additive mapping $T$ is called a two-sided centralizer in case $T$ is a left and a right centralizer. In case $T: R \longrightarrow R$ is a two-sided centralizer, where $R$ is a semiprime ring with extended centroid $C$, then there exists an element $\lambda \in C$ such that $T(x)=\lambda x$ for all $x \in R$ (see [4, Theorem 2.3.2]). An additive mapping $T: R \longrightarrow R$ is called a Jordan left centralizer if $T\left(x^{2}\right)=T(x) x$ holds for $x \in R$. In 1991, Zalar [34] proved that any Jordan left (respectively right) centralizer on a 2-torsion free semiprime ring is a left (respectively right) centralizer. Later [24], Molńar proved that in case we have an additive mapping $T: A \longrightarrow A$, where $A$ is a semiprime $H^{*}$-algebra, satisfying the relation $T\left(x^{3}\right)=T(x) x^{2}$ (respectively $T\left(x^{3}\right)=x^{2} T(x)$ ) for all $x \in A$, then $T$ is a left (respectively right) centralizer. For more related results concerning centralizers on rings and algebras we refer the reader to [13, 29-32], and [33], and references therein.

Let $R$ be a ring with involution. Following [3], an additive mapping $T: R \longrightarrow$ $R$ is said to be a left $*$-centralizer (respectively reverse left $*$-centralizer) if $T(x y)=$ $T(x) y^{*}$ (respectively $\left.T(x y)=T(y) x^{*}\right)$ holds for all $x, y \in R$. The definition of a right $*-$ centralizer (respectively reverse right $*$-centralizer) should be self explanatory. An additive mapping $T: R \longrightarrow R$ is called a $*$-centralizer if $T$ is both a left and right $*$-centralizer. An additive mapping $T: R \longrightarrow R$ is said to be a Jordan left $*$-centralizer if $T\left(x^{2}\right)=T(x) x^{*}$ is satisfied for all $x \in R$. Note that for some fixed element $a \in R$, the mapping $x \mapsto x^{*}$ is a reverse left $*$-centralizer and $x \mapsto a x^{*}$ is a reverse right $*$-centralizer on $R$. In Sections 2 and 3 , we study left $*$-centralizers and Jordan left $*$-centralizers in prime and semiprime rings. In particular, we prove that if a prime ring $R$ with involution admits an additive map $T: R \rightarrow R$ satisfying the relation $T\left(x^{m+n+1}\right)=\left(x^{*}\right)^{n} T(x)\left(x^{*}\right)^{m}$ for all $x \in R$, then there exists $q \in Q_{r}(R)$ such that $T(x)=q x^{*}$ for all $x \in R$, where $m$ and $n$ be positive integers such that $\operatorname{char}(R)=0$ or $m+n+1 \leq \operatorname{char}(R)$ (Proposition 2.3 and Theorem 2.4). Moreover, besides proving some results related to Jordan left $*$-centralizers (Theorem 3.4 and Theorem 3.5), we characterize normal rings among all noncommutative prime rings with involution (Theorem 3.1).

Let $S$ be a nonempty subset of $R$. A mapping $f: R \longrightarrow R$ is called a centralizing on $S$ if $[f(x), x] \in Z(R)$ for all $x \in S$. In particular, when $[f(x), x]=0$ for all $x \in S$, 
$f$ is said to be commuting on $S$. The fundamental result on commuting mappings and related mappings is due to Posner [27] who proved that if a prime ring $R$ admits a nonzero derivation $d$ such that $[d(x), x] \in Z(R)$ for all $x \in R$, then $R$ is commutative. This result was subsequently refined and extended by number of algebraists, we refer the reader to [5], [6], [21], and [22] for a comprehensive bibliography. Recently, some authors studied these notions in the setting of rings with involution (see [1], [25], and [26] and references therein). In 2014, Ali and Dar [2] obtained a *-version of Posner's second theorem. Precisely, they proved that if a prime ring with involution such that $\operatorname{char}(R) \neq 2$ and $d(S(R) \cap Z(R)) \neq(0)$ admits a nonzero derivation $d: R \rightarrow R$ satisfying $\left[d(x), x^{*}\right] \in Z(R)$ for all $x \in R$, then $R$ is commutative. In Section 4 , we review a few important results for generalized derivation and remove the condition $d(S(R) \cap Z(R)) \neq(0)$ (Theorem 4.1). Further, we give some examples which demonstrate that the restrictions imposed on the hypothesis of the various results are not superfluous.

\section{Preliminary results}

In this section, we collect some well known results and review a few important facts about right Martindale ring of quotients (see [23]) that will be needed in the subsequent discussions. For an extended work related to the theory of rings of quotients, we refer the reader to the book [4]. Let $R$ be a prime ring with right and symmetric Martindale ring of quotients $Q_{r}(R)$ and $Q_{s}(R)$, extended centroid $C$, and central closure $R_{c}=R C$, respectively. When $R$ has an involution, then we can extend to it an involution on $R_{c}$ and $Q_{r}(R)$ (see [8] and [23] for details) and hence in this case we may assume that it is an involution of $C$ as well. In the view of [4, Proposition 2.1.7], we state some properties of $Q_{r}(R)$ as follows:

(i) $R \subseteq Q_{r}(R)$;

(ii) For every $q \in Q_{r}(R)$ there exists a nonzero $*$-ideal $I$ of $R$ such that $I q \subseteq R$;

(iii) If $q \in Q_{r}(R)$ and $I$ is a nonzero $*$-ideal of $R$ such that $I q=0$, then $q=0$;

(iv) If $I$ is an $*$-ideal of $R$ and $f: I \longrightarrow R$ is a right $R$-module map, then there exists $q \in Q_{r}(R)$ such that $f(x)=q x^{*}$ for all $x \in I$.

We begin our discussion with the following results which are motivated by the work of Hvala [18].

Proposition 2.1. Let $R$ be a prime ring with involution and $f: R \longrightarrow R$ be a nonzero additive map satisfying $f(x y)=f(x) y^{*}$ for all $x, y \in R$. Then $R$ is commutative and there exists $\lambda \in C$ such that $f(x)=\lambda x^{*}$ for all $x \in R$.

Proof. By the assumption, we have $f(x y)=f(x) y^{*}$ for all $x, y \in R$. Computing $f(x y z)$ in two different ways, we obtain $f(x)\left[y^{*}, z^{*}\right]=0$ for all $x, y, z \in R$. This implies $f(R) R[R, R]=(0)$. Since $f$ is nonzero and $R$ is prime, we see that $R$ is commutative. Consequently, a map $g: R \rightarrow R$ defined by $g(x)=f(x)^{*}$ is an $R$-bimodule homomorphism (i.e., a centralizer). Hence, there exists $\mu \in C$ such that $g(x)=\mu x$ and so $f(x)=\lambda x^{*}$ for all $x \in R$, where $\lambda=\mu^{*}$. This proves the proposition completely.

Proposition 2.2. Let $R$ be a semiprime ring with involution and $f: R \longrightarrow R$ be an additive map satisfying $f(x y)=f(y) x^{*}$ for all $x, y \in R$. Then there exists $q \in Q_{r}(R)$ such that $f(x)=q x^{*}$ for all $x \in R$.

Proof. Note that a map $g: R \rightarrow R$ defined by $g(x)=f(x)^{*}$ is a right $R$-module homomorphism (i.e., a right centralizer). Hence, in the view of property $(i v)$ of $Q_{r}(R)$ just mentioned above there exists $\mu \in Q_{r}(R)$ (see [4, Proposition 2.1.7] for more details) such that $g(x)=\mu x$ for all $x \in R$. Thus, $f(x)=q x^{*}$, where $q=\mu^{*}$, for all $x \in R$. 
Proposition 2.3. Let $R$ be a 2-torsion free semiprime ring with involution. If $f: R \rightarrow R$ is an additive map such that $f\left(x^{2}\right)=f(x) x^{*}$ for all $x \in R$, then there exists $q \in Q_{r}(R)$ such that $f(x)=q x^{*}$ for all $x \in R$.

Proof. Let us define a map $g: R \rightarrow R$ by $g(x)=f(x)^{*}$. Then $g\left(x^{2}\right)=x g(x)$ for all $x \in R$. Hence, Proposition 1.4 in [34] implies that $g(x y)=x g(y)$ for all $x, y \in R$. Consequently, $f(x y)=f(y) x^{*}$ for all $x, y \in R$. Now, Proposition 2.2 yields the conclusion.

Having established the auxiliary results we can pass to our first theorem.

Theorem 2.4. Let $m$ and $n$ be positive integers, and $R$ be a prime ring with involution such that $\operatorname{char}(R)=0$ or $m+n+1 \leq \operatorname{char}(R)$. Let $T: R \rightarrow R$ be an additive mapping satisfying the relation $T\left(x^{m+n+1}\right)=\left(x^{*}\right)^{n} T(x)\left(x^{*}\right)^{m}$ for all $x \in R$. Then there exists $q \in Q_{r}(R)$ such that $T(x)=q x^{*}$ for all $x \in R$.

Proof. By the given hypothesis, we have $T\left(x^{m+n+1}\right)=\left(x^{*}\right)^{n} T(x)\left(x^{*}\right)^{m}$ for all $x \in R$. Applying the involution on both sides of the last expression, we obtain $T\left(x^{m+n+1}\right)^{*}=$ $x^{m} T(x)^{*} x^{n}$ for all $x \in R$. Define a new map $S: R \rightarrow R$ such that $S(x)=T(x)^{*}$ for all $x \in R$. Then $S$ is additive, since $T$ is additive. Therefore we have $S\left(x^{m+n+1}\right)=x^{m} S(x) x^{n}$ for all $x \in R$. Hence in the view of [[14], Theorem 3], we are forced to conclude that $S$ is a two sided-centralizer that is, $S(x y)=x S(y)=S(x) y$ for all $x, y \in R$. This implies that $(T(x y))^{*}=x(T(y))^{*}=(T(x))^{*} y$ for all $x, y \in R$. Again applying involution on both sides of the last expression, we find that $T(x y)=T(y) x^{*}=y^{*} T(x)$ for all $x, y \in R$. In the view of Proposition 2.2, we get the required result. The proof is now completed.

\section{Jordan left $*$-centralizers on prime rings}

We shall start our investigation with the following theorem which is motivated by the result proved in [7].

Theorem 3.1. Let $R$ be a noncommutative prime ring with involution such that char $(R) \neq$ 2. Then the following conditions are equivalent:

(i) $R$ is normal.

(ii) There exists a nonzero commuting Jordan left *-centralizer map $T$ on $R$.

Proof. Suppose (i) holds, that is, $R$ is a normal ring. Then the mapping $x \mapsto x^{*}$ is a commuting nonzero Jordan left $*$-centralizer on $R$. Now suppose (ii) holds, we want to prove $R$ is normal. Since $(i i)$ holds, so there exists a nonzero Jordan left $*$-centralizer $T: R \rightarrow R$ such that $[T(x), x]=0$ for all $x \in R$. Thus in the view of [[6], Theorem 3.2], there exists $\mu \in C$ and a map $\nu: R \rightarrow C$ such that

$$
T(x)=\mu x+\nu(x)
$$

for all $x \in R$. On the other hand, it follows from the proof of Proposition 2.3 that there exists $q \in Q_{r}(R)$ such that $T(x)=q x^{*}$ for all $x \in R$. Hence, we have $q x^{*}-\mu x \in C$ for all $x \in R$. Consequently, we conclude that

$$
\left[q x^{*}-\mu x, y\right]=0 \text { for all } x, y \in R .
$$

Since (3.1) is the identity involves involution, so it is a functional identity or the so-called $g$-identity (see [[4], Chapter 6]). In the view of [4, Theorem 6.4.6], we conclude that $\left[q x^{*}-\mu x, y\right]=0$ for all $x, y \in Q_{s}(R)$, the symmetric ring of quotients. Note that $Q_{s}(R)$ has the identity element 1 . Replacing $x$ by 1 in the above expression and using the fact that $\mu \in C$, we obtain $[q, y]=0$ for all $y \in Q_{s}(R)$. Thus,

$$
T(x)=\lambda x^{*}
$$

for all $x \in R$, where $\lambda=q \in C$. Since $T \neq 0$, it follows that $\lambda \neq 0$. Hence we conclude that $0=[T(x), x]=\left[\lambda x^{*}, x\right]=\lambda\left[x^{*}, x\right]$ for all $x \in R$. Thus, by the primeness of $R, R$ is normal. This proves the theorem completely. 
The above theorem has the following interesting consequences:

Corollary 3.2. Let $R$ be a prime ring with involution such that $\operatorname{char}(R) \neq 2$. Let $T$ be a nonzero Jordan left $*$-centralizer on $R$ such that $[T(x), x]=0$ for all $x \in R$. Then there exists $\lambda \in C$, the extended centroid of $R$ such that $T(x)=\lambda x^{*}$ for all $x \in R$.

Proof. The proof follows from the above theorem.

Remark 3.3. Here it will be interesting to see that if we consider an involution of the second kind in Theorem 3.1, then $R$ will be commutative. In Section 5, an example is also provided which shows that involution being of the second kind is a necessary condition. In fact, we prove the following result.

Theorem 3.4. Let $R$ be a prime ring with involution of the second kind such that $\operatorname{char}(R) \neq$ 2. If $R$ admits a nonzero Jordan left $*$-centralizer $T: R \rightarrow R$ such that $[T(x), x]=0$ for all $x \in R$. Then $R$ is commutative.

Proof. By Theorem $3.1 R$ is normal and since the involution of the second kind then $R$ is commutative by [2, Lemma 2.1].

The following example shows that the restriction of the second kind involution in above theorem is not redundant.

Example 3.5. Let $R=\left\{\left(\begin{array}{ll}a & b \\ c & d\end{array}\right) \mid a, b, c, d \in Z\right\}$. Clearly, $R$ is a prime ring with matrix addition and matrix multiplication. Define $*: R \longrightarrow R$ such that

$$
\left(\begin{array}{ll}
a & b \\
c & d
\end{array}\right)^{*}=\left(\begin{array}{cc}
d & -b \\
-c & a
\end{array}\right) \text { for all }\left(\begin{array}{ll}
a & b \\
c & d
\end{array}\right) \in R \text {. }
$$

Obviously, $Z(R)=\left\{\left(\begin{array}{cc}a & 0 \\ 0 & a\end{array}\right) \mid a \in Z\right\}$. Then $x^{*}=x$ for all $x \in Z(R)$, and hence $Z(R) \subseteq$ $H(R)$, which shows that the involution is of the first kind. If we consider $T(x)=x^{*}$, then $T$ is a Jordan left $*$-centralizer and $T$ satisfies $[T(x), x]=0$ for all $x \in R$. However, $R$ is not commutative. This shows that the hypothesis of second kind involution is essential in Theorem 3.4.

Theorem 3.6. Let $R$ be a noncommutative prime ring with involution such that char $(R) \neq$ 2. Let $T_{1}, T_{2}$ be two nonzero Jordan left $*$-centralizers on $R$ such that $T_{1}(x) x-x T_{2}(x)=0$ for all $x \in R$. Then there exists $\lambda \in C$ such that $T_{1}(x)=T_{2}(x)=\lambda x^{*}$ for all $x \in R$ and hence $R$ is normal.

Proof. By the given hypothesis, we have

$$
T_{1}(x) x-x T_{2}(x)=0
$$

for all $x \in R$. On linearizing (3.2), we get

$$
T_{1}(x) y+T_{1}(y) x-x T_{2}(y)-y T_{2}(x)=0
$$

for all $x, y \in R$. Replacing $y$ by $y x$ in (3.3) and using Proposition 2.2 we arrive at

$$
T_{1}(x) y x+T_{1}(x) y^{*} x-x T_{2}(x) y^{*}-y x T_{2}(x)=0
$$

for all $x, y \in R$. Using (3.2) in (3.4), we obtain $T_{1}(x) y x+T_{1}(x) y^{*} x-T_{1}(x) x y^{*}-y T_{1}(x) x=$ 0 . This can be further written as $\left[T_{1}(x), y\right] x+T_{1}(x)\left[y^{*}, x\right]=0$ for all $x, y \in R$. In the view of Proposition 2.3, $T_{1}(x)=q_{1} x^{*}$ (where $q_{1} \in Q_{r}(R)$ ) for all $x \in R$. Thus, we obtain

$$
\left[q_{1} x^{*}, y\right] x+q_{1} x^{*}\left[y^{*}, x\right]=0 \text { for all } x, y \in R .
$$

The identity (3.5) is a $g$-identity (see [4, Chapter 6]). In the view of [4, Theorem 6.4.6], we conclude that $\left[q_{1} x^{*}, y\right] x+q_{1} x^{*}\left[y^{*}, x\right]=0$ for all $x, y \in Q_{s}(R)$, the symmetric ring of 
quotients. Note that $Q_{s}(R)$ has the identity element 1 . Replacing $x$ by 1 in (3.5), we see that $\left[q_{1}, y\right]=0$ for all $y \in Q_{s}(R)$. Hence,

$$
T_{1}(x)=\lambda x^{*}
$$

for all $x \in R$, where $\lambda=q_{1} \in C$. Since $T_{1} \neq 0$, it follows that $\lambda_{1} \neq 0$. Also $T_{2}(x)=q_{2} x^{*}$, by Proposition 2.3. Hence from (3.2), $\lambda x^{*} x-x q_{2} x^{*}=0$ for all $x \in R$. The last identity is a $g$-identity. Thus by [4, Theorem 6.4.6], we obtain $\lambda x^{*} x-x q_{2} x^{*}=0$ for all $x \in Q_{s}(R)$, the symmetric ring of quotients. Replacing $x$ by 1 in last expression, we see that $\lambda=q_{2}$. Thus $T_{2}(x)=T_{1}(x)=\lambda x^{*}$ for all $x \in R$, where $0 \neq \lambda \in C$. Hence we conclude that $0=T_{1}(x) x-x T_{2}(x)=\lambda x^{*} x-x \lambda x^{*}=\lambda\left(x^{*} x-x x^{*}\right)$ for all $x \in R$. Thus by the primeness of $R, R$ is normal. This proves the theorem completely.

\section{Generalized derivations on prime rings}

Let $S$ be a nonempty subset of $R$. Following [2], a mapping $f: S \rightarrow R$ is said to be *-centralizing on $S$ if $\left[f(x), x^{*}\right] \in Z(R)$ for all $x \in S$, and is said to be *-commuting on $S$ if $\left[f(x), x^{*}\right]=0$ holds for all $x \in S$. The study of such mappings was initiated by Ali and Dar in [2]. Moreover, they characterized these mappings in prime rings with involution. Further they obtained a *-version of Posner's second theorem. Precisely, they proved that if a prime ring with involution such that $\operatorname{char}(R) \neq 2$ and $d(S(R) \cap Z(R)) \neq(0)$ admitting a nonzero derivation $d: R \rightarrow R$ satisfying $\left[d(x), x^{*}\right] \in Z(R)$ for all $x \in R$, then $R$ is commutative. Thus in the view of this result, it is natural to ask whether the analogue of the above mentioned theorem holds for generalized derivations. Our next theorem answers this question.

Theorem 4.1. Let $R$ be a prime ring with involution of the second kind such that char $(R) \neq$ 2. If $R$ admits a nonzero generalized derivation $F: R \rightarrow R$ such that $\left[F(x), x^{*}\right] \in Z(R)$ for all $x \in R$, then $R$ is commutative.

First we establish the following result.

Theorem 4.2. Let $R$ be a prime ring with involution of the second kind such that char $(R) \neq$ 2. If $R$ admits a nonzero left centralizer $T: R \rightarrow R$ such that $\left[T(x), x^{*}\right] \in Z(R)$ for all $x \in R$, then $R$ is commutative.

Proof. Suppose on the contrary that $R$ is not commutative. Since $T$ is a left centralizer of $R$ i.e., $T(x)=a x$ for all $x \in R$, where $a \in Q_{m r}(R)$. Note that $\left[T(x), x^{*}\right] \in Z(R)$ for all $x \in R$. This further implies that $\left[T(x)^{*}, x\right] \in Z(R)$ for all $x \in R$. Now using similar arguments as we used in Theorem 3.4 with necessary variation, we get the required result.

Now we are ready to give

Proof of Theorem 4.1. Suppose $R$ is not commutative. By the given assumption $\left[F(x), x^{*}\right] \in Z(R)$ for all $x \in R$. This further implies that $\left[F(x)^{*}, x\right] \in Z(R)$ for all $x \in R$. Therefore in the view of Brešar's result [6, Theorem A], there exists $\lambda \in C$ and an additive map $\mu: R \rightarrow C$ such that

$$
F(x)=\lambda x^{*}+\mu(x)
$$

for all $x \in R$. By [19, Theorem 4], there exists a derivation $d: R \rightarrow Q_{m r}(R)$ and $b \in Q_{m r}(R)$ such that $F(x)=d(x)+b x$ for all $x \in R$. So

$$
d(x)=\lambda x^{*}-b x+\mu(x)
$$

for all $x \in R$. Choose $0 \neq \beta^{*}=-\beta \in Z(R)$. Let $x \in R$. Then by (4.1), we have

$$
d(\beta x)=-\beta \lambda x^{*}-\beta b x+\mu(\beta x)
$$


for all $x \in R$. Also we can write

$$
\begin{aligned}
d(\beta x) & =\beta d(x)+d(\beta) x \\
& =\beta \lambda x^{*}-\beta b x+\beta \mu(x)+d(\beta) x
\end{aligned}
$$

for all $x \in R$. Comparing (4.2) and (4.3), we obtain

$$
2 \beta \lambda x^{*}+d(\beta) x=\mu(\beta x)-\beta \mu(x)
$$

for all $x \in R$. Commuting above relation with $x^{*}$ gives

$$
\left[d(\beta) x, x^{*}\right]=0
$$

for all $x \in R$. In the view of Theorem $4.2, d(\beta)=0$. This reduces (4.4) into

$$
2 \beta \lambda x^{*}=\mu(\beta x)-\beta \mu(x)
$$

for all $x \in R$. Thus $2 \beta \lambda x^{*} \in C$ for all $x \in R$, implying that $\lambda=0$. Hence, $F(x) \in Z(R)$ for all $x \in R$ and so $F=0$ which is a contradiction. This proves the theorem.

Example 4.3. Let $R=\left\{\left(\begin{array}{ll}a & b \\ c & d\end{array}\right) \mid a, b, c, d \in Z\right\}$. Clearly, $R$ is a prime ring with matrix addition and matrix multiplication. Define $*: R \longrightarrow R$ same as in Example 3.5. If we consider $F$ to be the identity map, then $F$ is a generalized derivation with an associated derivation $d=0$ and also a left centralizer, and $F$ satisfies the identity $\left[F(x), x^{*}\right]=0$ for all $x \in R$. However, $R$ is not commutative. This shows that the hypothesis of second kind involution is crucial in Theorem 4.1 and 4.2 .

The next example shows that it is essential for $R$ to be prime in the hypotheses of Theorem 4.1.

Example 4.4. Define $\mathcal{R}=R \times \mathbb{C}$, where $R, F$, and $*$ are same as in Example 4.3. Obviously, $\mathcal{R}$ is a semiprime ring with involution $\tau$, which is defined by $\tau(r, z)=\left(r^{*}, \bar{z}\right)$, where $\bar{z}$ denotes the complex conjugate of $z$. One can easily observe that $\tau$ is an involution of the second kind. Moreover, if $\mathcal{F}=(F(r), 0)$, then the hypothesis of Theorem 4.1 are satisfied but $\mathcal{R}$ is not commutative.

Acknowledgment. The authors are grateful to the referee(s) for his/her careful reading of the manuscript and improving Theorem 3.1. Also, we wish to express our sincere thanks to Professor Andre Leroy for his useful discussions during the preparation of the manuscript.

\section{References}

[1] S. Ali, On generalized *-derivations in *-rings, Pales. J. Math. 1, 32-37, 2012.

[2] S. Ali and N.A. Dar, On *-centralizing mappings in rings with involution, Georgian Math. J. 21 (1), 25-28, 2014.

[3] S. Ali, N.A. Dar, and J. Vukman, Jordan left *-centralizers of prime and semiprime rings with involution, Beitr. Algebra Geom. 54, 609-624, 2013.

[4] K.I. Beidar, W.S. Martindale III, and A.V. Mikhalev, Rings with generalized identities, Dekker, New York-Basel-Hong Kong, 1996.

[5] H.E. Bell and W.S. Martindale III, Centralizing mappings of semiprime rings, Canad. Math. Bull. 30 (1), 92-101, 1987.

[6] M. Brešar, Centralizing mappings and derivations in prime rings, J. Algebra 156, 385-394, 1993.

[7] M. Brešar and J. Vukman, On some additive mappings in rings with involution, Aequationes Math. 38, 178-185, 1989.

[8] C.L. Chaung, *-differential identities of prime rings with involution, Tran. Amer. Math. Soc. 316 (1), 251-279, 1989. 
[9] V. De Filippis, Posner's second theorem and an annihilator condition with generalized derivations, Turkish J. Math. 32 (2), 197-211, 2008.

[10] V. De Filippis and M.S. Tammam El-Sayiad, A note on Posner's theorem with generalized derivations on Lie ideals, Rend. Semin. Mat. Univ. Padova 122, 55-64, 2009.

[11] B. Dhara and S. Ali, On n-centralizing generalized derivations in semiprime rings with applications to $C^{*}$-algebras, J. Algebra Appl. 11 (6), 1250111, 2012.

[12] B. Dhara and V. De Filippis, Notes on generalized derivations on Lie ideals in prime rings, Bull. Korean Math. Soc. 46 (3), 599-605, 2009.

[13] M. Fošner and J. Vukman, A characterization of two-sided centralizers on prime rings, Taiwan J. Math. 11, 1431-1441, 2007.

[14] M. Fošner and J. Vukman, An equation related to two-sided centralizers in prime rings, Rocky Mountain J. Math. 41 (3), 765-776, 2011.

[15] O. Golbasi and E. Koc, Notes on commutativity of prime rings with generalized derivation, Commun. Fac. Sci. Univ. Ank. Ser. A1-Math. Stat. 58 (2), 39-46, 2009.

[16] I.N. Herstein, Jordan derivations on prime rings, Proc. Amer. Math. Soc. 8, 11041110, 1957.

[17] I.N. Herstein, Rings with Involution, University of Chicago Press, Chicago, 1976.

[18] B. Hvala, Generalized derivations in rings, Comm. Algebra 26, 1147-1166, 1998.

[19] T.K. Lee, Generalized derivations of left faithful rings, Comm. Algebra 27 (8), 40574073, 1999.

[20] F.A. Lopez, G.E. Rus, and S.E. Campos, Structure theorem for prime rings satisfying a generalized identities, Comm. Algebra 22 (5), 1729-1740, 1994.

[21] J. Mayne, Centralizing automorphisms of prime rings, Canad. Math. Bull. 19, 113$117,1976$.

[22] J. Mayne, Centralizing mappings of prime rings, Canad. Math. Bull. 27, 122-126, 1984.

[23] W.S. Martindale III, Prime rings satisfying generalized polynomial identities, J. Algebra 12, 574-584, 1969.

[24] L. Molńar, On centralizers of an $H^{*}$-algebra, Publ. Math. Debrecen 46, 89-95, 1995.

[25] L. Oukhtite, A. Mamouni, Generalized derivations centralizing on Jordan ideals of rings with involution, Turkish J. Math. 38 (2), 225-232, 2014.

[26] L. Oukhtite, S. Salhi, and L. Taoufiq, Generalized derivations and commutativity of rings with involution, Beitr. Algebra Geom. 51 (2), 345-351, 2010.

[27] E.C. Posner, Derivations in prime rings, Proc. Amer. Math. Soc. 37, 27-28, 1988.

[28] N. Rehman and V. De Filippis, Commutativity and skew-commutativity conditions with generalized derivations, Algebra Colloq. 17 , 841-850, 2010.

[29] I. Kosi-Ulbl and J. Vukman, On centralizers of standard operator algebras and semisimple $H^{*}$-algebras, Acta Math. Hung. 110, 217-223, 2006.

[30] J. Vukman, Centralizers in prime and semiprime rings, Comment. Math. Univ. Carolin. 38, 231-240, 1997.

[31] J. Vukman, Centralizers on semiprime rings, Comment. Math. Univ. Carolin. 42, 237-245, 2001.

[32] J. Vukman and I. Kosi-Ulbl, Centralizers on rings and algebras, Bull. Austral. Math. Soc. 71, 225-234, 2005.

[33] J. Vukman and I. Kosi-Ulbl, On centralizers of semiprime rings with involution, Stud. Sci. Math. Hungar. 43, 77-83, 2006.

[34] B. Zalar, On centralizers of semiprime rings, Comment. Math. Univ. Carolin. 32, 609-614, 1991. 